\title{
ESTACIONES TERMALES EUROPEAS. ENTRE LA CIUDAD Y EL TERRITORIO
}

Mònica BATALLA FARRÉ

La singularidad del territorio termal confiere a las estaciones termales unas cualidades especiales y las convierte en enclaves atractivos a lo largo de la historia. Algunos balnearios europeos fueron grandes atractores turísticos durante el siglo XIX. En consecuencia, importantes desarrollos arquitectónicos y urbanísticos se llevaron a cabo en ellos. Determinadas piezas específicas de estas ciudades balneario, a menudo ubicadas en el límite entre la ciudad y el territorio, fueron claves para dichos desarrollos y dan las pautas para entender la estructura urbana de aquellas ciudades y la relación con su territorio y paisaje.

Estaciones termales, turismo, territorio, paisaje

\section{EUROPEAN THERMAL TOWNS}

\section{Between the city and the territory}

The uniqueness of the thermal territory gives to the thermal towns special qualities and turns them into attractive enclaves throughout history. Some European spa towns were strong tourism attractors during the 19th century. Accordingly, great architectural and urban developments were implemented on them. Some pieces of these spa towns, often located at the boundary between the city and the territory, are key to these developments and give guidelines for understanding the urban structure of these cities and the relationship with its territory and landscape.

Thermal towns, tourism, territory, landscape 


\section{SINGULARIDAD DEL TERRITORIO TERMAL}

La posición de las ciudades balneario está muy determinada en el territorio. Sólo las podremos encontrar en enclaves que cumplen unas características específicas (accidentes geográficos o fracturas tectónicas, con presencia de fuentes termales); lugares donde las aguas de escorrentía aprovechan las fracturas para ascender y aparecer en superficie.

Si ubicamos las ciudades balneario en los mapas físico y tectónico de Europa (fig. $n^{\circ} 1 y^{\circ} 2$ ) podemos observar cómo éstas se concentran en los accidentes geográficos -Pirineos, Macizo central Europa, Alpes, Apeninos, Peninos y Cambrianos (Reino Unido), Cárpatos y Balcanes- con presencia de fallas.

Por ello, podemos considerar el territorio termal como un soporte agrietado, en el que estos núcleos de población se ubican en los puntos de presencia de aguas termales.

Según las diferentes posiciones en el territorio, la composición y temperatura de las aguas varían (aguas frías o calientes, cálcicas o sulfurosas, etc.), por lo que cada territorio termal tiene calidades y características ambientales determinadas. Estos territorios tendrán también características paisajísticas diferentes y específicas. Ambas confieren a cada territorio termal un carácter singular que lo distingue del resto.

\section{CIUDADES BALNEARIO EN EL TERRITORIO TERMAL}

Las diferentes localizaciones de los centros termales en referencia a la ciudad también determinarán la relación de estos centros -así como de las propias ciudades- con el territorio y su paisaje.

En una primera clasificación podemos diferenciar modos distintos de construcción de la ciudad termal en relación con sus centros urbanos.

- Balneario aislado

- Balneario periférico

- Balneario en núcleo urbano 

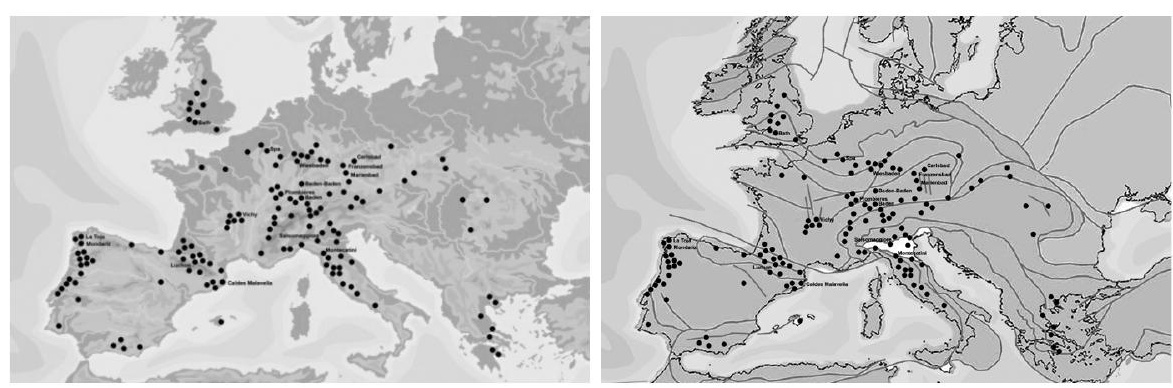

Fig.1 y 2: Superposición de ciudades termales en Europa (fuente: Moldoveanu, 1999). Mapa físico de Europa (fuente: CNICE) y International Tectonic Map of Europe (fuente: Comission for the Geological Map of the World. UNESCO). Elaboración propia.

\section{Balneario aislado}

La mayoría de estos centros balnearios aislados se encuentran situados en parajes singulares, apartados de los núcleos urbanos. Su relación con el núcleo urbano a través de vías de comunicación permite que el propio recorrido de acceso al balneario se convierta en un modo de reconocimiento del territorio.

A menudo estos centros termales propiciarán la llegada de vías ferroviarias a los territorios donde se encuentran, junto con estaciones o apeaderos vinculados al uso del centro termal, que se convierten en piezas determinantes para la configuración del espacio entorno al centro balneario.

En los espacios exteriores de estos centros balnearios se planifican los recorridos, visuales, ambientes de sol y sombra y vegetación. Esta ubicación permite una mayor relación del balneario con el entorno natural, que se caracteriza en la mayoría de casos por cambios topográficos fuertes y vegetación frondosa.

\section{Balnearios periféricos}

Podemos considerar balnearios periféricos aquellos situados próximos a las ciudades termales y que se caracterizan por generar espacio urbano a su alrededor, en continuidad con la trama urbana.

Estos centros balnearios también motivarán la llegada de la red ferroviaria a las localidades en las que se encuentran.

\section{Balnearios en núcleo urbano}

En este caso los centros termales y sus espacios exteriores se ven inmersos y acotados por la trama urbana histórica y su relación con el centro de la ciudad es mucho más directa y activa. 
En las tres tipologías de balneario los espacios exteriores, paseos o parques serán de gran importancia. En general, el máximo uso de la terapia termal se dará en la estructura del verde urbano de las ciudades balneario, que a la vez será el eje principal de articulación de la vida urbana.

\section{EL TERRITORIO TERMAL COMO GENERADOR DE ESPACIOS TURÍSTICOS}

Coincidiendo con el renacimiento italiano y los primeros viajes de humanistas curiosos de Italia y la antigüedad, se empieza a denotar un cierto interés por la visita a estos centros termales (Montaigne, 1581) y por la experiencia de las aguas vinculada a un espacio arquitectónico.

Tras un periodo de declive, en el siglo XVII se iniciará en Europa una etapa de recuperación del interés por los centros termales, que tendrá su máximo esplendor entremediados del siglo XVIII y mediados del siglo XIX, propiciado por la evolución de las técnicas médicas, el discurso higienista y la mejora de la accesibilidad territorial.

El Grand Tour, en el que jóvenes aristócratas de principios del siglo XVIII recorrían Europa Occidental durante uno o dos años con la intención de distinguirse y convertirse en gentlemen (Boyer, 2002: 14), incluía la visita a algunos centros termales. Después del Grand Tour, los enclaves termales empiezan a atraer un número considerable de visitantes, sobretodo aristocracia y burguesía.

Ya entrado el siglo XVIII, algunos personajes británicos relevantes proponen prácticas de ocio en lugares de reconocido prestigio, lo que Boyer (2002: 14) califica de "revolución turística». Así pues, en el caso de Beau Nash en Bath, podemos empezar a hablar de un cierto concepto de turismo vinculado al termalismo, en el que pacientes se entremezclan con turistas en busca del ocio que ofrecerán estas ciudades como complemento a la oferta de los centros termales. De esta forma, el «turismo» se convierte en el motor de desarrollo de estas ciudades balneario.

Los siglos XVIII y XIX se caracterizaron por el gran desarrollo arquitectónico y urbanístico de las ciudades termales europeas, ya que se convirtieron en fuertes polos de atracción de visitantes. Esta mayor afluencia significó la necesidad de mayores inversiones en hoteles, parques o paseos y otras distracciones, como casinos.

Los enclaves termales son distintos de otros destinos turísticos. Las ciudades balneario fueron lugares de encuentro y de identidad colectiva, aunque cada una de ellas intentó destacar por encima de las otras. Se pretendía mostrar un cierto "producto" que las hiciera atractivas, de forma que se promovieron 
como un bien de consumo. El marketing urbano se convirtió ya en el siglo XIX en determinante para su promoción.

Las actividades de ocio alrededor del tema del agua se convertirán en el reclamo de estas ciudades, que proponen una experiencia diferente a la vida cotidiana. Lo que las hizo atractivas no fueron sólo los centros de salud, sino también los equipamientos y servicios vinculados a éstos, el uso de los espacios exteriores colectivos.

Las ciudades balneario emergen y son construidas en torno al agua, la comercialización y relación de la ciudad con el producto como marca (Vichy o Carlsbad) y productos auxiliares (agua embotellada, jabones o sales de bańo, entre otros). El hecho termal dirige el desarrollo urbano de las ciudades balneario. El ambiente de las ciudades viene dictado por los centros termales: el producto y la marca, la creación de instalaciones, un tipo de industria hotelera, tiendas de lujo, etc.

Las ciudades balneario ofrecerán un mundo ilusorio, otra realidad recreada. Se usarán los mismos nombres para las instalaciones vinculadas (Grand Hotel, Park, Excelsior, Imperial, Palace), las mismas ofertas lúdicas y deportivas (casino, tenis y golf) y las mismas arquitecturas históricas eclécticas (Anderson, 2002).

Esto provocará que las ciudades balneario pierdan cierta identidad, con lo que buscarán nuevos argumentos que les permitan diferenciarse del resto. Un hecho distintivo de cada una de ellas serán las características propias de cada lugar, su geniusloci. Así pues, pondrán énfasis en las características exclusivas de su emplazamiento natural -montañas, lagos o saltos de agua- y cada ciudad hará publicidad de estas características para recuperar su singularidad.

En el siglo XIX se crearán nuevas zonas ajardinadas. También se promoverán los caminos en los alrededores, las visitas al campo y el acceso a geografías que dirigen a los visitantes a vistas y panoramas privilegiados del entorno natural de estas ciudades termales.

\section{INTERESES DE LA INVESTIGACIÓN}

En una primera aproximación y búsqueda de información de estudios existentes sobre ciudades balneario descubrimos que existen muchos artículos sobre temas médicos, sociales e históricos vinculados al fenómeno termal. También un cierto número de estudios artísticos y arquitectónicos, principalmente referidos a las edificaciones de las villas termales.

Tras el análisis de los artículos encontrados en las bases de datos de publicaciones científicas, hemos detectado un vacío de estudio en la relación 
de los centros termales, y por extensión de las ciudades balneario, con su territorio y paisaje. Aunque en general se hace referencia a la peculiaridad de las ciudades balneario en términos urbanísticos, existen pocos estudios urbanísticos que traten la relación de las ciudades balneario con el territorio y paisaje, o cómo los centros termales generan ciudad.

Se plantean algunas preguntas: ¿Cómo es el urbanismo en estas ciudades? ¿El "hecho termal» las predispone a un tipo de urbanismo específico? ¿Podemos hablar de urbanismo termal? ¿Cómo es la relación entre los centros termales, la ciudad y su territorio y paisaje? ¿Su ubicación concreta en el territorio es determinante en su configuración urbanística? ¿Esta gran afluencia de visitantes y nuevo modos de ocio vinculados al hecho termal provocan la generación de un tipo concreto de configuración urbanística? ¿Plantea esta configuración nuevos conceptos de definición de la ciudad?

Observamos como en las primeras guías de viajes de mediados del siglo XIX, referentes en la época, los paseos tienen un papel destacado. En las guías Murray del territorio inglés y francés aparece la clasificación Walks and Excursions, referidos tanto a paseos dentro de la ciudad como en el territorio. También en la guía Joanne Les Bains d'Europe; guide descriptif et médical de 1860 aparecen las promenades como una categoría descriptiva de las cerca de 200 villas termales europeas que están incluidas en el libro.

Encontramos de modo recurrente que el termalismo en las ciudades balneario se da sobre todo en los espacios abiertos de la ciudad. Por ello, el propio uso terapéutico de las ciudades balneario establece que el espacio público sea el soporte de la vida urbana. Se nombran repetidamente paseos, parques y jardines, lo que evidencia que éstos se han convertido en los elementos principales de desarrollo de la vida urbana.

Aparecen nuevas preguntas sobre cómo son estos espacios abiertos que soportan el uso diario de la ciudad balneario, si estos espacios públicos de las ciudades termales son diferentes del resto de ciudades contemporáneas o qué relación tienen con el territorio.

\section{POSICIÓN Y DESARROLLO DE LA CIUDAD BALNERIO EN EL TERRITORIO. BATH, VICHY, BADEN-BADEN}

La investigación analizará la evolución de las más destacadas ciudades balneario europeas durante los siglos XVIII y XIX. Se centrará inicialmente en Vichy, Bath y Baden-Baden, por ser ciudades balneario representativas de tres regiones termales históricas (Francia, Inglaterra y Bohemia) y que aparecen de modo recurrente en artículos de diversos autores expertos como Jarrassé (2002), Boyer (1996) y Moldoveanu (1999). 
En los tres casos, la ciudad permanece amurallada hasta el siglo XVIII. Si bien dentro de las murallas existen recintos destinados a los baños, las tres también cuentan con enclaves termales en sus alrededores, que en algunos casos generan la construcción de edificaciones vinculadas. Cuando se derriban las murallas, la relación de la ciudad con estos centros termales-sobre todo en Bath y Baden-Baden-tendrá una gran importancia en el desarrollo y expansión de estos centros urbanos hacia el territorio.

Podemos observar que cada una de estas ciudades tiene una manera diferente de abordar la estructura de los espacios libres. Mientras Vichy se basa en ejes de recorrido en el parque termal (y posteriormente en los largos paseos que recorren el Parc des Sorces), Bath estructura el verde urbano a través de grandes piezas de parque y Baden-Baden se centra en la relación con el territorio y el paisaje. En los tres casos, estos paseos y piezas de parque están en el límite entre la ciudad y el territorio.

La pieza del paseo se repite en todas ellas, aunque toma diversos nombres como promenade, walk o kolonnande. Los paseos no serán exclusivos de las ciudades balneario, sino que seguirán las corrientes urbanísticas contemporáneas de cada una de las regiones en las que se encuentran estas ciudades de acuerdo con los planteamientos sociales comunes en gran parte de Europa; es decir, las corrientes higienistas del siglo XIX, apoyadas en los Select Committee Public Walks de 1833 en Inglaterra y los planes de Haussmann y Napoleón III de mediados del siglo XIX para el territorio francés.

Si bien el paseo se inicia en la moda de las clases educadas inglesas del siglo XVIII como acción social, en el siglo XIX aparecen los paseos con mayor

Fig. 3: Vichy, siglo XV. Autor desconocido.

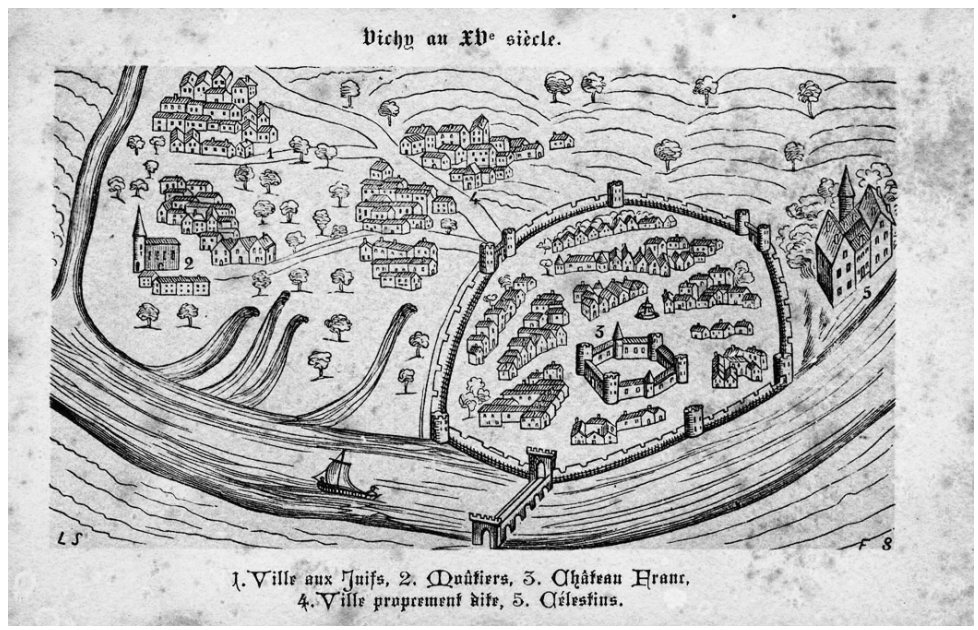




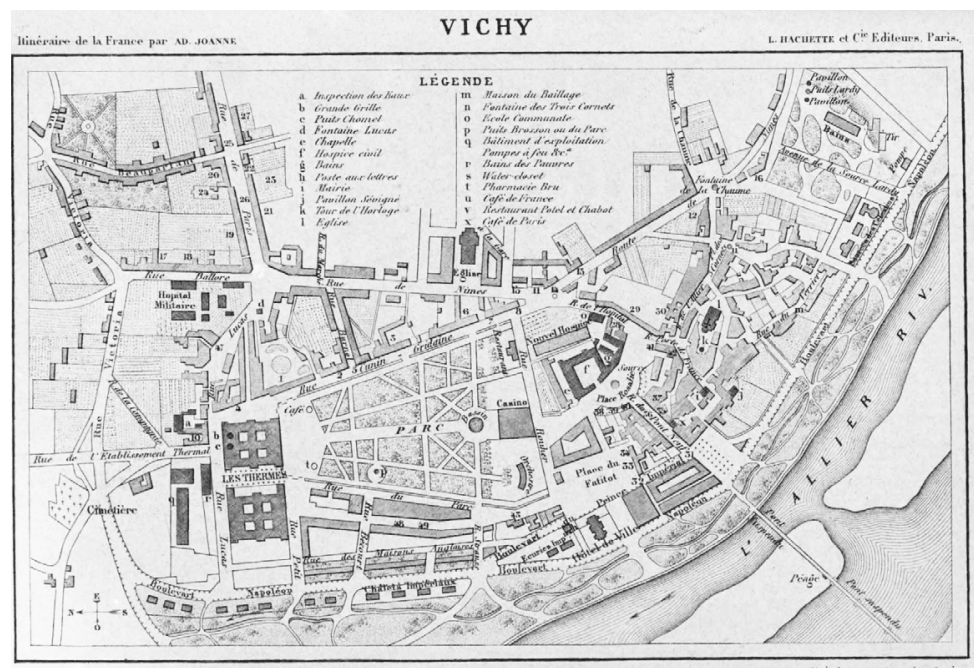

Fig. 4: Vichy et ses environs. Fuente: Autor desconocido, 1863, Guide Joanne.

contundencia -tendrán en las ciudades balneario su máximo esplendor-, que se convertirán en piezas clave del desarrollo de la vida urbana.

\section{Vichy}

Si partimos del análisis de su cartografía en el siglo XV, Vichy es una ciudad amurallada rodeada por zonas de explotación agrícola. Las fuentes termales están vinculadas al monasterio, que es el que ejerce el control de las aguas.

En el siglo XVIII se derriban las murallas y aparece el primer establecimiento termal sólo accesible para una minoría burguesa, que es la que tiene posibilidades de desplazamiento con carruajes privados. Vichy estaba incluido en los recorridos del Grand Tour.

A mediados del siglo XIX empiezan en Francia los proyectos de urbanización del segundo imperio de Napoleón III y la difusión del modelo de Haussmann para París (conjuntamente con el ingeniero y paisajista A. Alphand), donde se abren grandes avenidas y se determina una estructura del verde urbano. Este modelo se repetirá en todo el territorio francés.

Las características del modelo de verde urbano que se propone consisten en la abertura de grandes ejes de comunicación, la sistematización del verde urbano con la presencia de grandes piezas centrales y el saneamiento de las calles. Estas características serán también las que se propondrán para la ciudad de Vichy en el siglo XIX.

En el año 1853 se empieza en Vichy una política de reestructuración urbana, pero siempre vinculada al centro termal. Se mejorarán las instalaciones de 
los baños, aparecerán hoteles, quioscos comerciales o cafés-restaurante, todos ellos relacionados con los espacios exteriores y la vida termal de la ciudad.

En 1870 se da el mayor cambio urbanístico en Vichy, impulsado por Napoleón III, para el embellecimiento general de la estación termal. Se propone la creación de rutas de agua, salas de espectáculos o la llegada del Chemin de Fer. El cambio más importante es la ampliación y adecuación de los espacios verdes alrededor del río Allier para propiciar el paseo y el acceso al Parc des Sources, terreno baldío hasta aquel momento.

El higienismo se sitúa en el centro del debate y las actuaciones responden a la voluntad de hacer más salubre la ciudad de Vichy, de modo que sus habitantes copien los modelos de actuación para los espacios privados.

Por ello, aunque a mediados del siglo XIX los paseos son un elemento común en Francia, la ciudad de Vichy se configura como un modelo para otras ciudades balneario: Napoleón III pone a Vichy en el centro de las estaciones termales europeas.

El Parc des Sources y el parque a orillas del río Allier serán el lugar de encuentro tanto para los clientes estacionales de la estación termal como para los habitantes locales. En ellos se creará la vida urbana de Vichy; serán espacios propicios para disfrutar del «tiempo libre».

Fig. 5: Vichy, 1899. Grabado por John Bartholomew \& Co.

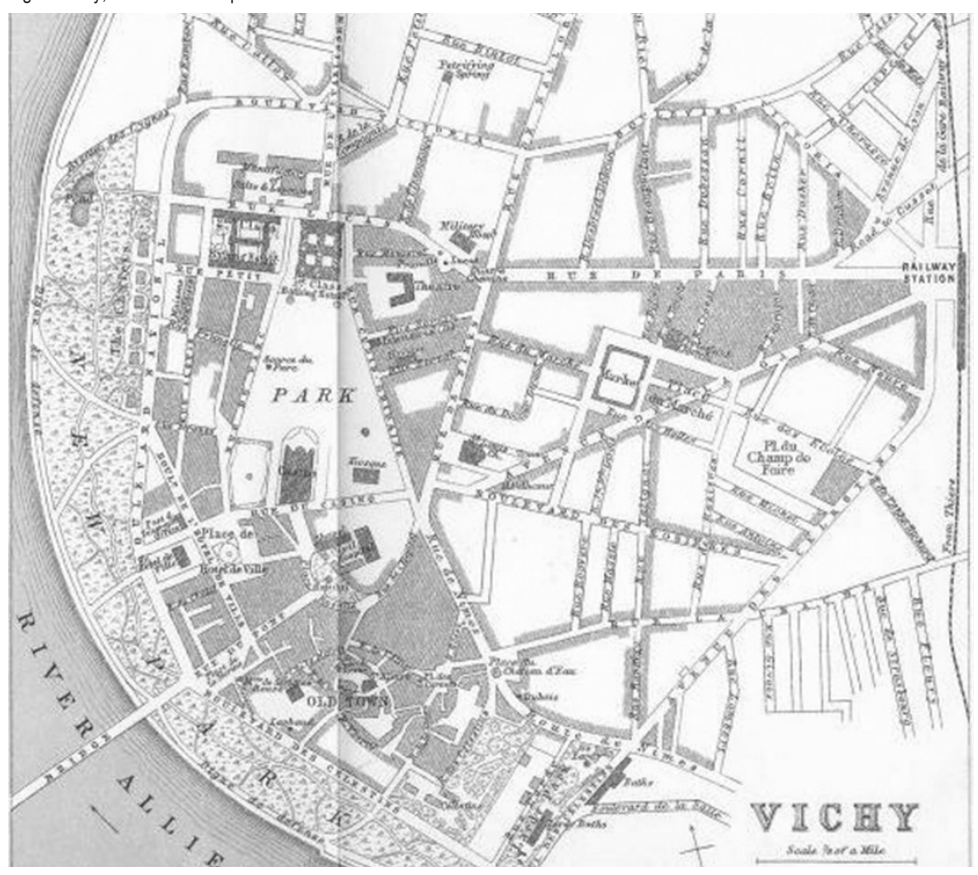


El Parc des Sources es el destino final del paseo, de modo que se convierte en un recorrido médico para llegar al pulmón verde de la ciudad, que es el parque (Dutheil, 2006). El parque y el paseo se transforman en «lugares» de la ciudad, no solamente un espacio de naturaleza recreada; serán los puntos neurálgicos de la vida de la ciudad.

El paseo y el parque se elevan al rango de actividad cultural dentro de la ciudad y en ellos se darán lugar diferentes actividades de entretenimiento. Ambos se convierten en el espacio social por excelencia de la ciudad de Vichy.

\section{Bath}

En Inglaterra los paseos se conocerán como walks y aparecen por primera vez en Tunbridge Wells, hacia 1638. Posteriormente, en los inicios del siglo XVIII el Mall de Londres será lugar de reunión de la gente de moda. A lo largo del siglo XVIII malls, parades, walks, promenades y esplanades serán el equivalente en el exterior de los assemblyrooms, nombre con el que en Inglaterra e Irlanda se denominan las estancias a cubierto destinadas a las clases burguesas para la reunión y el entretenimiento.

Los publicwalks se extenderán rápidamente en toda Inglaterra. Fuera de Londres fueron especialmente populares en spas y resorts, aunque no exclusivamente en éstos; cualquier lugar con pretensiones de tener una sociedad educada casi inevitablemente debía tener un lugar donde sus miembros pudieran caminar conjuntamente.

En el siglo XVII los parques serán mayoritariamente lugares destinados a actividades recreacionales, como la caza, pero empezarán a acoger algunos paseos para uso social. En el siglo XVIII los jardines y parques, que serán conocidos como pleasure gardens, ofrecerán otras actividades de ocio-música, refrescos, asientos confortables- de las que se podrá disfrutar previo pago de una entrada. La mayoría de estos jardines tendrán un paseo principal arbolado. A raíz de la importancia que empiezan a tomar los parques dentro del esquema de funcionamiento de la ciudad, se dota a Bath de los nuevos jardines de Spring, Grosvenor y Sydney Gardens.

A principios del siglo XVIII Bath experimenta sus primeras transformaciones urbanas (Feltham, 1813). El maestro de ceremonias de Bath, Beau Nash, junto con el arquitecto John Wood padre, son los encargados de transformar la ciudad, que todavía está rodeada por las murallas romanas, para dar respuesta al aumento de número de visitantes.

En 1708 Nash se encarga de crear el primer paseo en Bath. Estará ubicado en Orange Grove, junto a la abadía, y consiste en un paseo pavimentado de 200 pies de largo por 27 pies de ancho y flanqueado por sicómoros. 


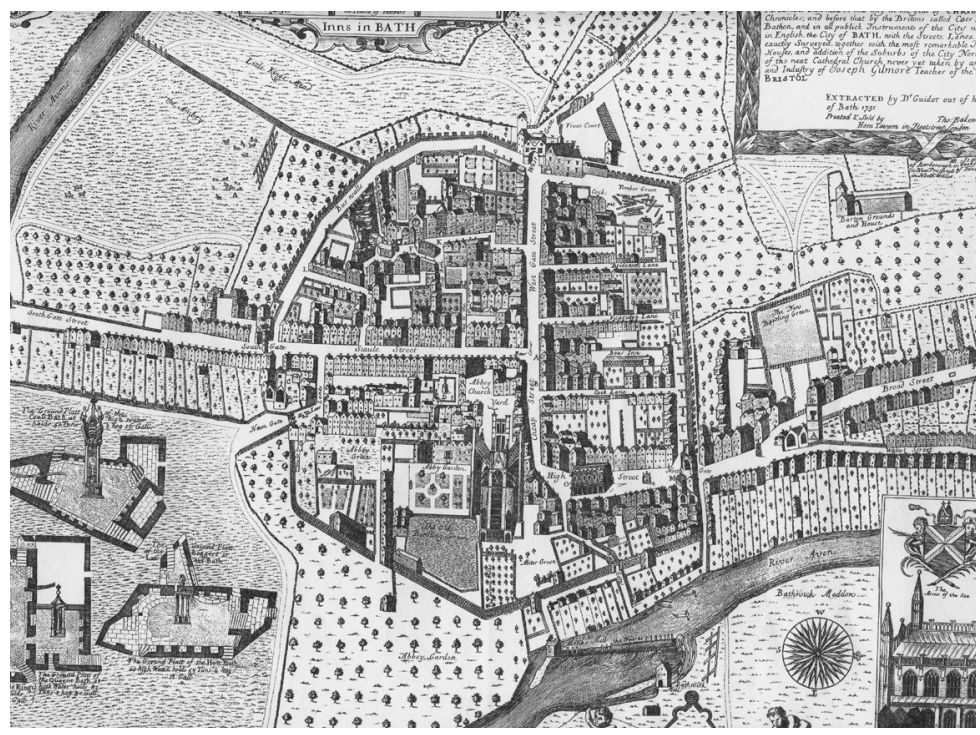

Fig. 6: Detail of The City of Bath. Fuente: Gilmore, 1694, Bath in Time-Bath Preservation Trust.

Hacia 1728 crea nuevas residencias con una nueva arquitectura, procurando la integración paisajística con la utilización de piedra local y la construcción de edificaciones de uso común. Se mejorará la calidad de las calles existentes, ensanchándolas y pavimentando las zonas donde se pretende que los visitantes paseen. Las calles serán las zonas comunes y los parques y jardines estarán reservados a aquellos visitantes que hayan pagado para acceder.

Entre 1740 y 1743 John Wood construye dos parades, norte y sur, de las cuales la North Parade será conocida como la Grand Parade. Tal como decía Girouard (1990): «Durante los siguientes cuarenta años más o menos, la Grand Parade y el Mall de Londres serán las dos más famosas promenades de Inglaterra. Las vistas desde la promenade hacia el campo, parque o el mar, se pusieron de moda en los pueblos ingleses, con un efecto radical en su naturaleza.»

En cualquier caso, Beau Nash procurará que la calidad del paseo sea excepcional ya que el hecho del pasear será una de las actividades que ocupará gran parte del tiempo de los visitantes.

No obstante, el uso de la ciudad y el acceso a los diferentes lugares de la ciudad están muy determinados para cada visitante. A su llegada a la ciudad, éstos deben pagar por aquellos servicios que querrán recibir durante su estancia y a los espacios que querrán acceder.

Nash iniciará un cierto condicionamiento de los espacios extra-muros de la ciudad de Bath, así como la mejora de las vías cercanas a la ciudad para facilitar 


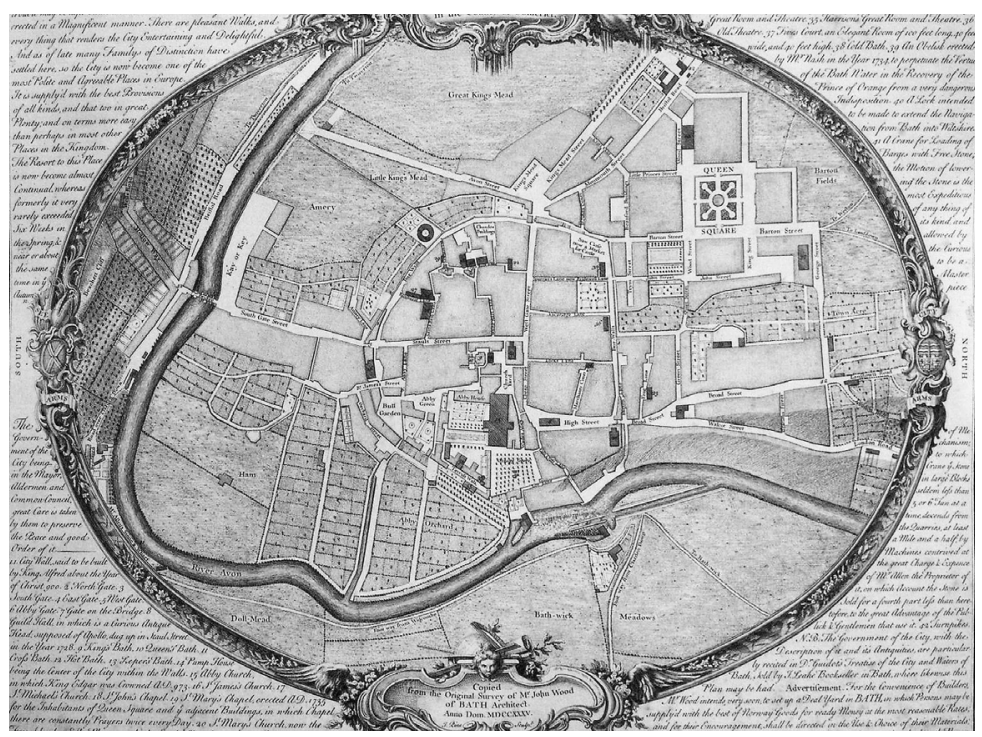

Fig. 7: A plan of the city of Bath. Fuente: John Wood, 1735, Bath in Time - Bath Central Library Collection.

la llegada de visitantes desde Londres. Por lo tanto, para Nash es importante tanto los espacios de dentro de la ciudad como los exteriores y sus conexiones.

La ciudad empieza a recibir gran cantidad de visitantes, ya que Nash ha conseguido que Bath sea considerada la más importante de las ciudades termales de moda del siglo XVIII.

Para permitir el crecimiento de la ciudad, entre 1755-1770 se derriban las murallas, lo que iniciará su verdadero desarrollo con proyectos de Wood padre e hijo. Sus proyectos marcarán la imagen que la ciudad de Bath tiene hoy en día, y de la que tomarán referencias formales otras ciudades balneario como Crescent o Circus, entre otras. Las calles nuevas se hacen conforme a las condiciones de salubridad, tamańo y servicios adecuados, mientras que las viejas se amplían.

Se proponen recorridos por el interior de la ciudad y por el paisaje del entorno, que se harán en carruaje. La visita al entorno forma parte también de la oferta lúdica de la ciudad.

Debido a los conflictos que generó la tarifación del servicio de carruajes -que se calculaba en millas de recorrido-, se ubicarán unos puntos de paradas en el territorio, a distancia calculada. Por lo tanto, en el caso de los viajeros en carruaje, el reconocimiento y percepción del entorno vendrán condicionados por la existencia de estas paradas. 


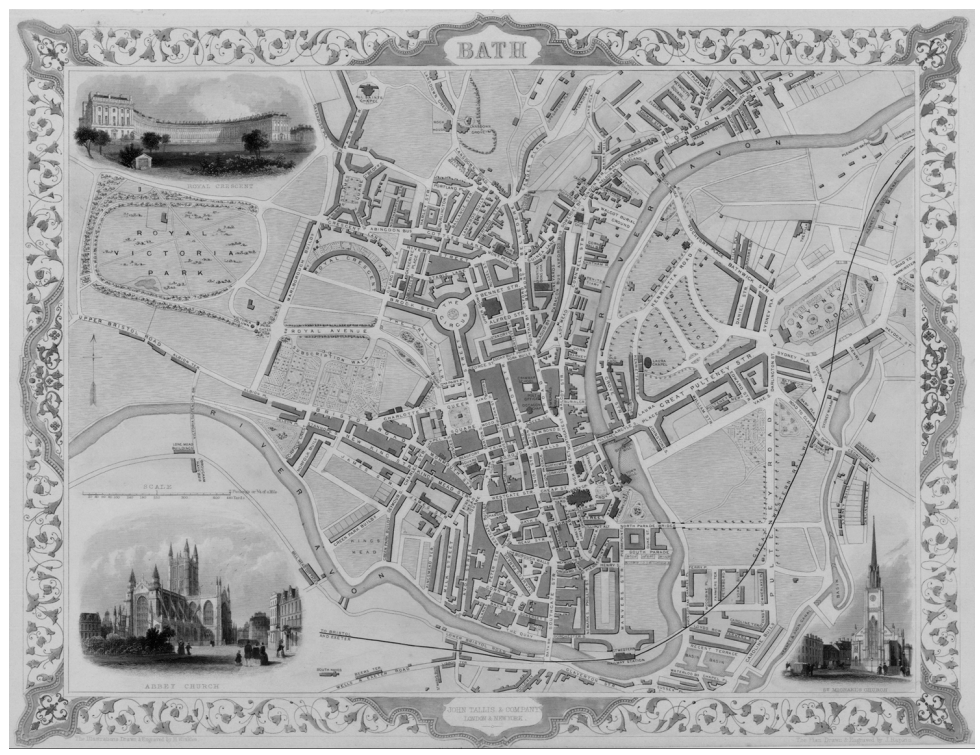

Fig. 8: Bath. Fuente: John Tallis, 1851. http://www.kingscourtgalleries.co.uk/

En 1833 se presenta en Inglaterra el informe del Parliamentary Select Committee on Public Walks, por el que se recomienda la creación de espacios abiertos, seguros y saludables en los alrededores de las ciudades muy pobladas, con el objetivo de crear espacios para el paseo y ejercicio y promover la salud y el confort entre sus habitantes. Hasta aquel momento las clases alta y media eran conscientes de la necesidad del uso del espacio exterior para el bienestar. Con el informe se propone hacer accesibles estos espacios abiertos y concienciar de su uso a las clases sociales más trabajadoras.

\section{Baden-Baden}

Baden-Baden sufrió en 1689 un gran incendio debido a la ocupación de las tropas francesas, lo que destruyó casi completamente la ciudad. No será hasta 1797, en el Rastatter Kongress, que será reconocida de nuevo por sus valores como ciudad termal. En 1765 se construye la PromenadeHaus, cuya pieza principal será la Kurhaus-Kolonnaden, paseo con tiendas tradicionales a cubierto y que conducía desde el establecimiento balneario al centro de la ciudad, acompañado por cuatro hileras de castaños.

A principios del siglo XIX se proponen planes urbanísticos para una nueva zona balneario, lo que favorecerá que en 1810 se desarrolle el Lichtentaler Allee. Éste se convertirá en el principal paseo arbolado, continuación de la PromenadeHaus, y seguirá el recorrido del río Oos hasta la ciudad. 


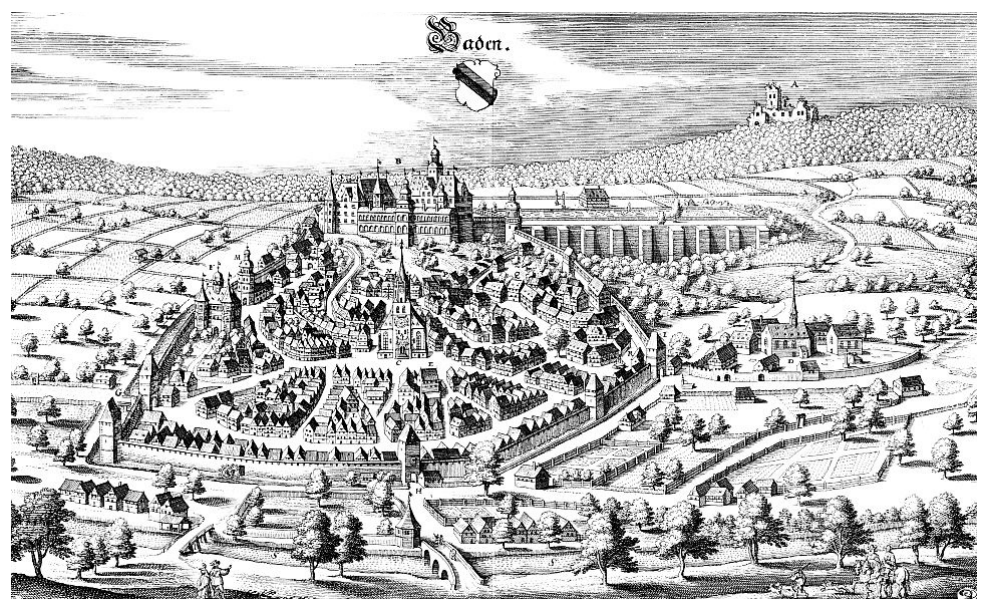

Fig. 9: Baden. Fuente: Matthäus Merian, 1643, http://www.vintage-maps.com/

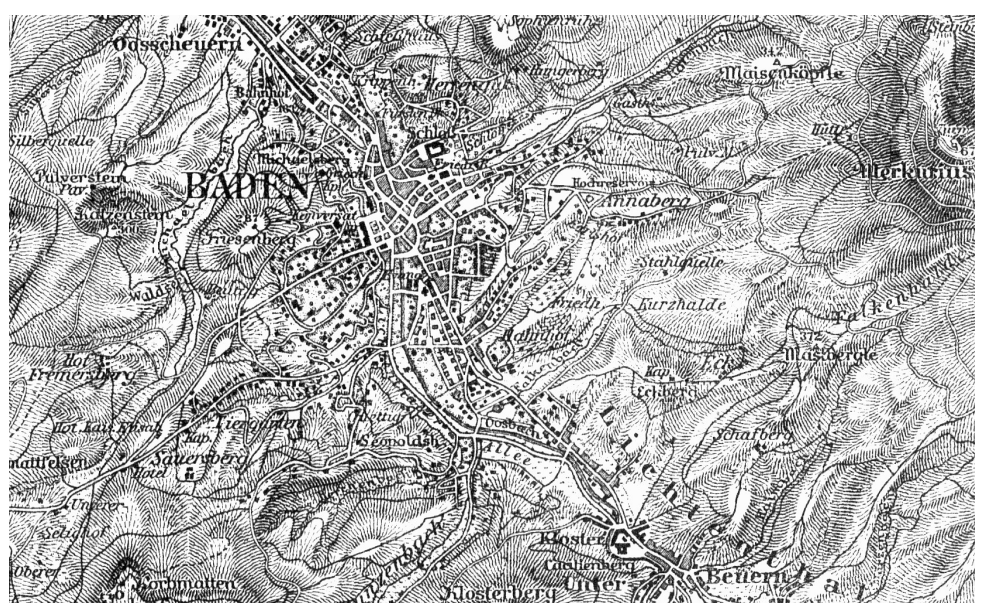

Fig. 10: Baden. Fuente:2 $2^{\mathrm{a}}$ mitad siglo XIX, Meyers Konversations-Lexikon.

Favorecida por la prohibición de los juegos de azar en París, la ciudad verá su máxima transformación a mediados del siglo XIX. Se plantearán diversos recorridos por la ciudad y algunos atravesarán las piezas del parque.

Siendo Baden-Baden referencia para otras ciudades balneario, los paseos con columnas conocidos como Kolonnaden se extenderán por todas las ciudades balneario de la Bohemia.

Desde la ciudad también se promoverán los recorridos por el entorno natural. El interés por el conocimiento de los espacios exteriores en Baden- 
Baden viene determinado por la existencia de ruinas y por la topografía, que condicionan la existencia de terrazas de cultivo de viña de alto valor paisajístico.

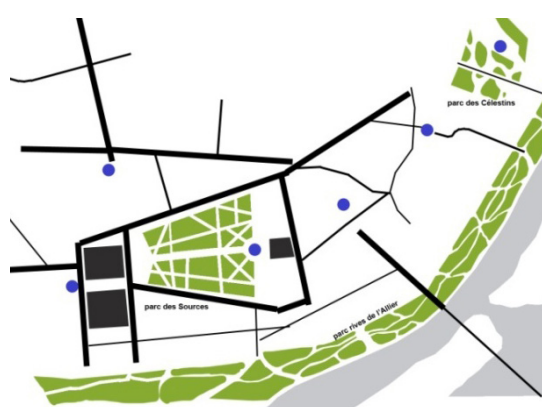

Fig. 11: Esquema de sistema de verdes y promenades, según el plano de Vichy incluido en la guía Joanne "Vichy et ses environs" 1863. Elaboración propia.

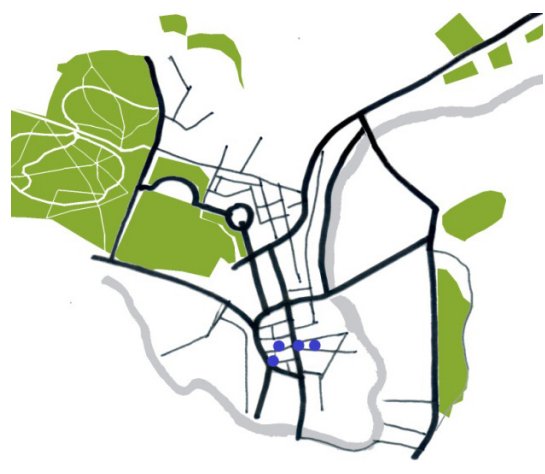

Fig. 12: Esquema de sistema de verdes y walks, siguiendo el mapa de Bath elaborado por Thomas Moulé 1836. Elaboración propia.

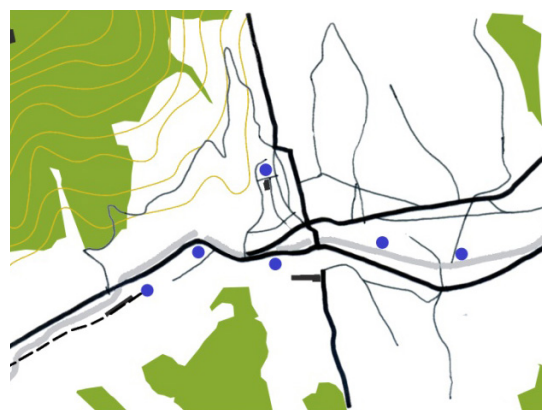

Fig. 13: Esquema de sistema de verdes y promenades. Según plano 1875 Baden-Baden, de autor desconocido. Elaboración propia.

\section{Vichy}

Recorridos lineales

Ciudad balneario que crece periférica al centro urbano generando nueva ciudad.

El paseo o promenade es el soporte del desarrollo de la vida urbana.

\section{Bath}

Grandes piezas de parques

Modelo de ciudad balneario en núcleo urbano.

«La alternancia de espacio verde y espacio construido, la disimulación del paisaje construido como paisaje virgen, la multiplicidad de vistas sobre el campo son todo factores que crearán la estética de su paisaje» (Zanni, N. 1993).

\section{Baden-Baden}

Vinculación territorial

Modelo de ciudad balneario en núcleo urbano.

Será el recorrido hacia el entorno natural de la ciudad el que generará el contacto con el paisaje local propio del lugar. 


\section{CONCLUSIONES}

La relación que se establece entre las diferentes piezas principales en la ciudad termal -el balneario, los equipamientos vinculados, los paseos y el parque termal- es la que estructurará la ciudad balneario.

La determinada posición de estas piezas de la ciudad termal, ubicadas de modo recurrente en las zonas de contacto entre la ciudad y el territorio, dará soluciones concretas para la resolución de los límites del tejido urbano en las ciudades balneario.

La existencia de enclaves termales extramuros en la ciudad medieval generará, una vez se derriban las murallas, que la relación de las ciudades con éstos determine un tipo de crecimiento y desarrollo característicos de la ciudad termal.

Los paseos y parques en la ciudad termal serán piezas indispensables para el desarrollo de la actividad cotidiana de la ciudad balneario. De manera repetida, los paseos y las piezas de parque están a caballo entre la ciudad y el territorio, lo que las sitúa como piezas clave en la resolución de sus límites, de comprensión de sus paisajes y de crecimiento de las ciudades balneario.

Las ciudades balneario hacen una apuesta rotunda por el paseo, que se convierte en el elemento clave en la configuración del espacio público. El parque termal se configura como un elemento central en las ciudades balneario en cuanto que es también soporte de sus paseos. 


\section{Bibliografía}

General

ANDERSON, S.C., TABB, B. (2002). "Water, Leisure and Culture: European Historical Perspectives. (Leisure, Consumption and Culture)". Oxford/New York : Berg.

BOYER, M. (1999). "Historie du tourisme de masse". Paris: Presses Universitaires de France.

BOYER, M. (2002). "El turismo en Europa, de la edad moderna al siglo XX'. En Turismo y nueva sociedad (13-31). País Vasco: Revista Historia Contemporánea, 25.

CORBIN, A. (1988). "Le territoire du vide. L'Occident et le desir du rivage, 1750-1840'. París: Aubier, collection historique, $411 \mathrm{p}$. ISBN: 2-7007-2217-5.

JARRASSÉ, D. (2002). "La importancia del termalismo en el nacimiento y desarrollo del turismo en Europa en el siglo XIX'. En Turismo y nueva sociedad (33-49). País Vasco: Revista Historia Contemporánea, 25.

FERRÉ, J.S. (2000). "Panorama actual de las aguas minerales y minero-medicinales en España". Ponencia: Historia de los balnearios en España. Arquitectura-Patrimonio-Sociedad. Madrid: Instituto Tecnológico GeoMinero de España.

MOLDOVEANU, M et al. (1999). "Ciudades termales en Europa”. Barcelona: Lunwerg Editores.

MONTAIGNE, M. (1774). "Journal du voyage de Michel de Montaigne en Italie, par la Suisse \& l'Allemagne en 1580 \& 1581". París : chez Le Jay.

PIE NINOT, R. y ROSA JIMÉNEZ C. J. (2010). “Turismo líquido”. Málaga: Universidad de Málaga y Escuela Técnica Superior de Arquitectura de Málaga.

SOLÀ-MORALES, J. M et al. (1986). "Arquitectura balneària a Catalunya". Barcelona: Generalitat de Catalunya/ Cambra de la Propietat Urbana de Barcelona.

Vichy

ALPHAND, A. (1867). "Les promenades de Paris, 2 vols". Paris: J. Rothschild, vol. 1873.

DUTHEIL, F. (2006). "Promenadedans les parcs de Vichy et saisons thermales (1850-1870)", Ethnologie française, Vol. 36, p. 543-552. DOI : 10.3917/ethn.063.0543.

JAMOT, C. (2001). "Vichy : du tourisme à la ville, de la ville au tourisme / Vichy : links betweentourism and the town". In: Géocarrefour. Vol. 76 n², . pp. 133-138.

PINOL, J.L. (2003). "Histoire de l'Europe urbaine" De l'Antiquité au XVIlle siècle. Paris : Éditions du Seuil, cop.

Bath

FELTHAM, J. (1813). "Guide to all the Watering and Sea-Bathing Places ; with a description of the lakes; a sketch of a tour in Wales; and itineraries", Longman.

GIROUARD, M (1990). "The English Town" Yale University Press.New Haven and London: Ed. Pothecary. MELVILLE, L. (1907). "Bath under Beau Nash" London: E. Nash. 
HALL, C.M. (2002). "The geography of tourism and recreation: Environment, place and space". PAGE, Stephen. Psychology Press.

ZANNI, N. (1993). "L'immagine della città termale". Milano: Edizioni Angelo Guerini e Associati. 